\title{
Alveolar repair with equine xenogen biomaterial: case report
}

\begin{abstract}
The alveolar ridge is the bone around the root portion of the tooth. Various environmental and physiological effects accompany or disassemble, influence their fullness and performance. As such, its production as well as its conservation is linked to a constant presence of the tooth. Bone resorption in the socket after dental extraction is an undesirable natural and physiological occurrence, which often makes it difficult to place a dental implant in the selected position. The use of bone grafts has the function of creating a matrix in which the coagulated blood will form and enable the healing process. Xenografts are biomaterials of animal origin, they are deproteinized totally eliminating the organic component and avoiding immunogenicity. The present study aimed to evaluate the influence of equatorial graft on the speed of technical repair as preparation for rehabilitation with osseointegrated implants. This paper presents a case report of a patient who underwent tooth extraction, with immediate placement of equine xenograft and radiographic follow-up for recent days. Bone of equine origin when reproduced in bovine origin and ovine was the one that most showed similarity with human bone tissue, in relation to visual roughness, also related to porosity. Through radiographic follow-up, it was possible to evaluate if the equine xenograft is effective in bone neoformation in the socket after extraction and grafting. Further research should be performed with computed tomography to better evaluate the efficacy of equine xenograft
\end{abstract}

Keywords: extraction, xenograft, bone formation
Volume I I Issue I - 2020

\author{
Bruna de Souza Eleutério da Silva,' Priscila \\ Carneiro Oliveira,' Laís Sara Egas, ${ }^{2}$ Roberta \\ Catapano Naves, ${ }^{3}$ Antonielson Costa \\ Coutinho' \\ 'Regional University Center of Brazil, UNIRB, Brazil \\ ${ }^{2}$ Faculty of Dentistry of Araçatuba, UNESP, Brazil \\ ${ }^{3}$ Bahiana Faculty of Medicine and Public Health, Brazil
}

Correspondence: Priscila Carneiro Oliveira, Regional University Center of Brazil, UNIRB, Brazil,Tel +55 7/98|4I6303, Email carneiro_priscil@hotmail.com

Received: November 28, 2019 | Published: January 23, 2020
Abbreviations: EPI's, individual protection equipment; LP, periodontal ligament; BMP's, bone morphogenetic proteins; CT, computed tomography

\section{Introduction}

Immediately after extraction, there are large changes in the height and width of the resident alveolar ridge, regardless of whether the extraction is just a single dental unit or multiple. Also, note that the proportion of bone resorption is higher in the buccal bone plate when used with the tongue / palate. ${ }^{1,2}$

In fact, once a tooth is extracted, about $25 \%$ of bone volume is lost within the first year after extraction. Over time, these changes cause resorption, may develop and help to occur. $40-60 \%$ loss of alveolar bone volume around five years. The consequent absence of bone in the alveolar crest is a result of the gradual loss of horizontal dimension followed by a slight vertical bone loss. ${ }^{3}$

Bone loss is a consequence of the sequelae of surgical trauma, as well as pathologies that affect both acute and chronic or because of severe periodontal disease. These bone defects are not removed alveolar, causing an insufficient bone volume, which leads to an impossibility or incorrect installation of the implant, the aesthetic point of view. ${ }^{4,5}$

Bone regeneration can be performed through three (3) different mechanisms: osteogenesis, osteoinduction and osteoconduction. Understand that osteogenesis has a bone self-producing feature, due to pressure from living cells that exist in it (osteoblasts), in addition to conserving bone production as well as conserving bone production. Osteoinduction is a bone neoformation through bone morphogenetic proteins (BMP's), a growth factor that hardly exists in bones. Osteoconduction is the process that separates a biological structure, or physical matrix, that serves as a framework for the deposition of new bone. ${ }^{6}$

The types of bone grafts used in dentistry are: autogenous, allogeneic, xenogeneic and alloplastic. Autogenous bone harvested from the patient forms new bone by osteogenesis, osteoinduction and osteoconduction. Allografts captured from cadavers have osteoconductive and possibly osteoinductive properties, but are not osteogenic. Xenografts and alloplastic bone materials are typically only grafted osteoconductors. ${ }^{5}$

At first, to reconstruct as bone lesions or surgeries used to graft type of autogen, because these were classified as gold standard, due to this type of graft originated in the individual, or that hinders its rejection. However, this approach began to demonstrate as disadvantage, the need for the second donor site, from these problems and the grafting utilities, survived other types of grafting in place of autogen, xenogens used by another species and In addition to allogen captured by bone banks (cadavers), these factors are also important, such as the risk that the organism will have a negative response and reject the material or disease transmission.

In the search to find a material that has not allowed, survive alloplastic grafts, use the laboratory environment. This graft has been increasingly accepted in the market because it has advantages such as ease of use and manipulation, its surgical time is reduced and it is 
also the type of material that is not extracted by the individual, or that reduces the risks of morbidity, because there is no need for two (2) surgical sites in the same individual. Although it has some advantages, it may occur the rejection of the organism to the biomaterial and, in some cases, after the infectious condition. ${ }^{8}$

Xenogen grafts are characterized by the fact that they are acquired through a different species from the individual receptor. They consist of inorganic parts of bone of animal origin. ${ }^{9,10}$ The equine graft has been shown to be satisfactory when used in human grafts, and has biocompatibility and revascularization, with angiogenesis. Within the limits of the study sample, it was found that equine material can be safely and successfully used to increase mandibular rearrangements. ${ }^{11}$

Di Stefano et al. ${ }^{12}$ through a study that aimed to evaluate a bone neoformation over a period, after increasing the maximum level with enzymatic equine bone graft. Perform a human research, 77 isolates, which are presented 4 (four) to 7 (seven) $\mathrm{mm}$ of resident alveolar bone. In conclusion in this study, we observed that when an equine bone is employed to increase the maximal level, a new bone formation occurs within a short period of time $(<3$ months), after a bone graft at the implant site can be safely performed. Between 3 (three) and 5 (five) months after maxillary volume surgery. ${ }^{12}$

Understanding and understanding the dimensional changes that occur in the alveolar process as a result of extraction, conditions the preference for atraumatic tooth extraction techniques, aiming at preserving the bone cortices of the alveolar process for later prosthetic treatment. ${ }^{13-16}$

As reports of studies with equine biomaterials are scarce, this study aimed to evaluate the influence of equine bone grafts on the speed of tissue repair after tooth extraction as preparation for rehabilitation with osseointegrated implants through a case report.

\section{Materials and methods}

This is a case study and for the present study was selected as an adult/young individual, with approximate weight between 60 and $70 \mathrm{~kg}$. The clinically healthy individual underwent basic clinical examinations to rule out the possibility of oral diseases present, prevention of surgical interventions and which may interfere with the results of this study.

The selected patient underwent bone graft surgery, without which type of Biogen ${ }^{\circledR}$ - Bioteck ${ }^{\circledR}$ biomaterial graft (Bioteck S.p.A VicenzaItaly). The anatomical site chosen for graft placement was the socket after extraction of the dental unit from the individual's arch. Graft surgery was performed with appropriate instrumental instruments and sterilized according to standardization recommended for the clinical case studied in this study. The entire surgical team made use of personal protective equipment (PPE) according to biosafety standards.

Patient E.L.S, 31 years old, female, leucoderma, compared to the outpatient clinic of oral rehabilitation clinic at the Regional University Center of Brazil - UNIRB, without dental course, complaining of "broken tooth". After anamnesis analysis it was found that one patient did not present any systemic alteration. He was then classified as an ASA 1 patient with good oral health. On radiographic examination, a residual root was found in unit 15 (Figure 1), indicating an extraction of the unit. It was proposed as treatment plan the rehabilitation of the unit with bone implants performed in two surgical times and filled with socket with equine graft, opting for the biomaterial Bio-Gen Bioteck ${ }^{\circledR}$.

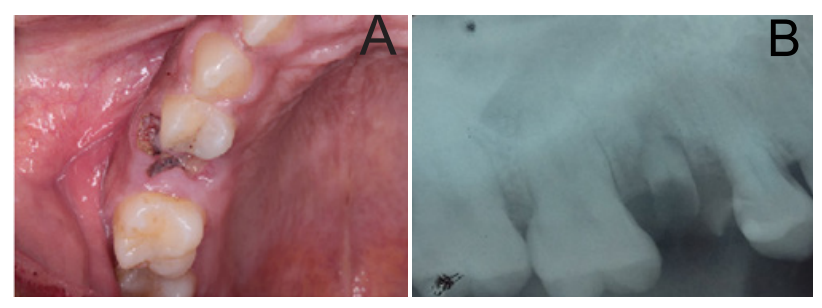

Figure I (A) Initial clinical aspect. (B) Initial radiographic appearance.

For extraction of the started unit, anesthesia with Lidocaine HCL 2\% + epinephrine 1: 100,000 were performed. Thus, infraorbital nerve block and papillary complementation were performed. Then, an intrasucular incision (selected 15C) was made, followed by detachment of the gingival tissue with the Molt No. 9 detacher (Figure 2). Immediately after detachment, initiate the procedure of periodontal ligament dislocation (LP) and disconnection of the root remnant, due to the need for minimal trauma for the conservation of the alveolar walls, try an atraumatic extraction of the units using the straight chelate period ${ }^{\circledR}$ (Figure 3 ). Next, the alveolus was curettable with Lucas's curette associated with abundant irrigation with saline for cleaning the alveolus.

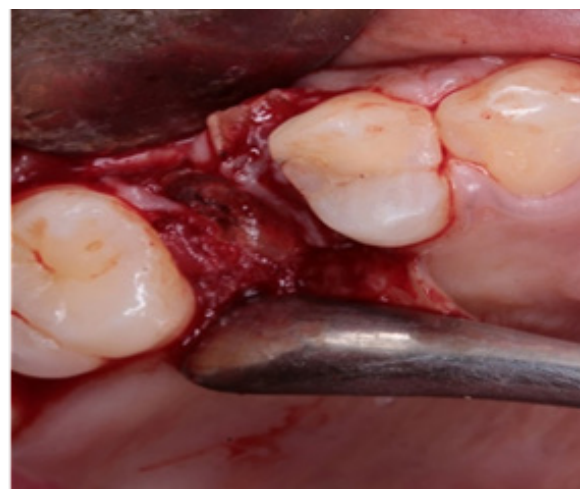

Figure 2 Appearance after tissue detachment.

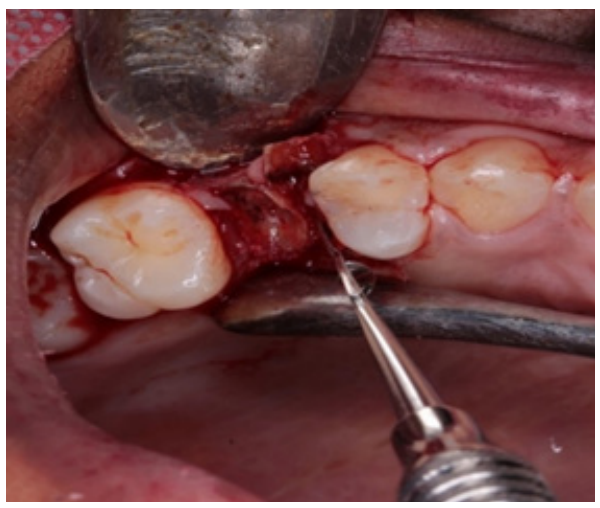

Figure 3 Dislocation and extraction of the periotome unit.

After extraction, no damage to the crystals was reported, and therefore, the preservation of the alveolar bone walls can be observed,

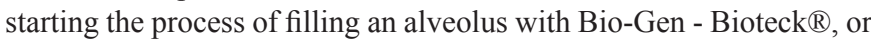
the biomaterial not inserted in the alveolus. And compressed with the aid of a calculator to ensure that all content is filled (Figure 4). Finally, a simple sample with shalon ${ }^{\circledR} 5-0$ absorbable suture was performed using only the gingival tissue. In this case, the use of membranes was not necessary (Figure 5). The patient underwent periapical radiographs 
for follow-up, after which he remained with a control of 07,30, 60 and 90 days (Figure 6).
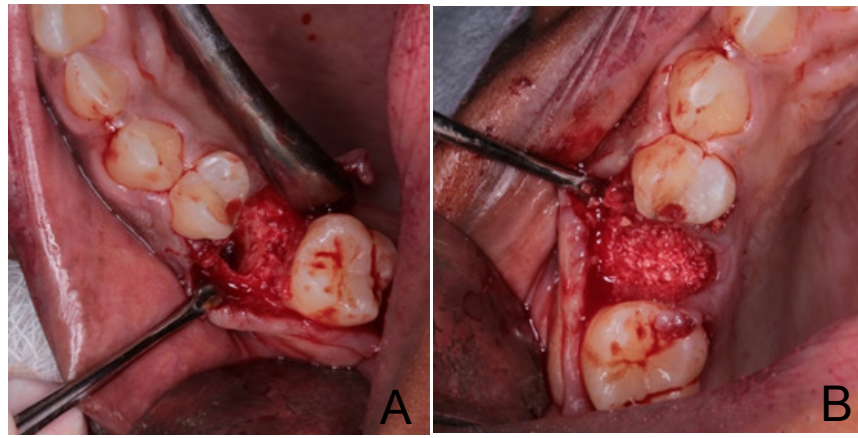

Figure 4 (A) preservation of the alveolar walls. (B) Alveolus filled with equine bone graft.

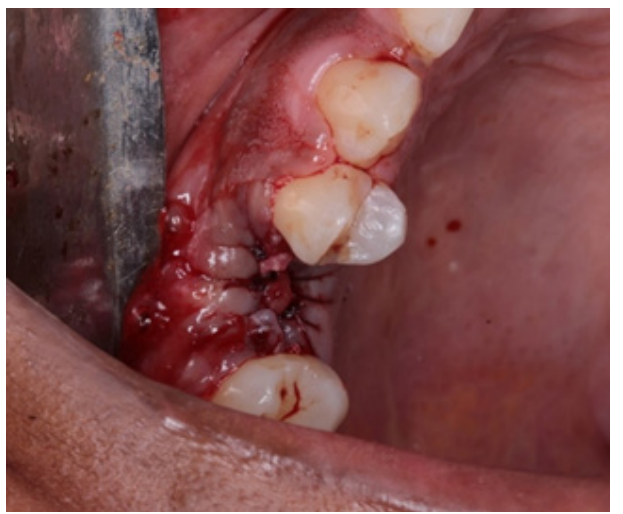

Figure 5 Synthesis and final surgical aspect.
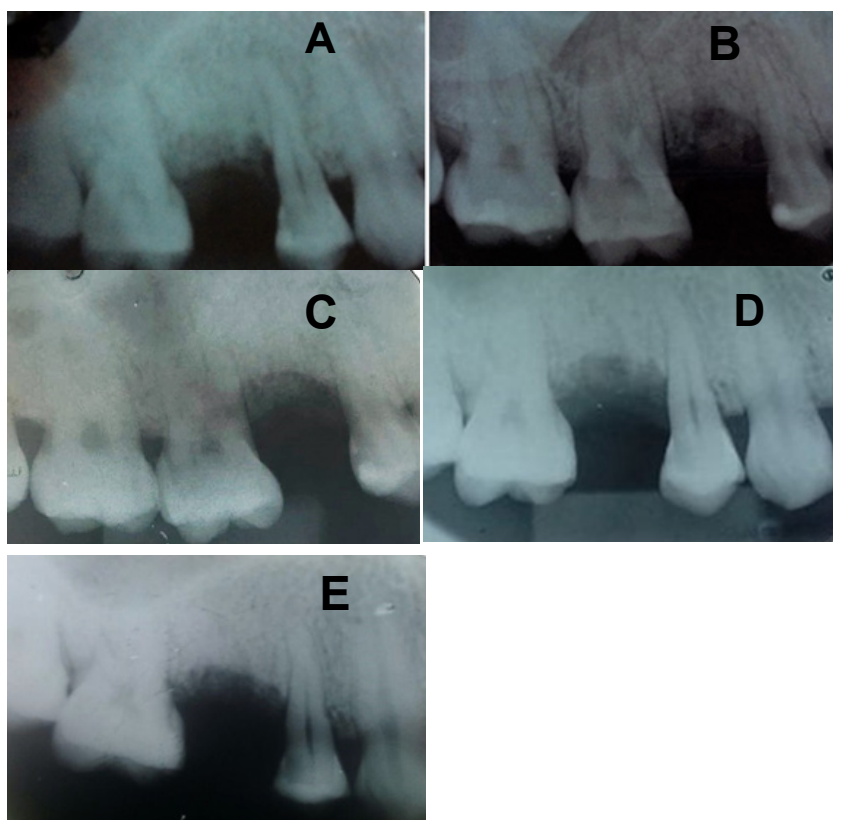

Figure 6 (A) Immediate periapical radiography. (B) Periapical control radiograph 07 days after bone graft. (C) Periapical control radiograph 30 days after bone graft. (D) Periapical control radiograph 60 days after bone graft. (E) Control periapical radiograph 90 days after bone graft.

\section{Results and discussion}

\section{Result}

Through the follow-up with periapical radiographs for 90 days, it was possible to evaluate that the new type of equine origin xenogen graft induces satisfactory bone neoformation and in a short period, it is possible to observe this new bone formation in the place where it was inserted. The equine xenograft, which makes it possible to install a subsequent dental implant with a good neoformation.

\section{Discussion}

The study by Araújo et al. ${ }^{14}$ corroborates by stating that the physiological resorption that happens in the alveolar bone after tooth extraction has been confirmed in experimental studies that attributed horizontal (width) and vertical (height) bone loss, which modifies the three-dimensional profile of the alveolar ridge and the availability. Of bone required for implant installation. ${ }^{4}$

Zafiropoulos et al. ${ }^{17}$ stated that the preference for implant rehabilitation has increased and to be performed it is necessary to preserve the alveolar bone in the place where the implant will be installed and to preserve the gingival contour properly, especially in the aesthetic regions. These requirements must be considered and planned before and during dental extraction, and for this purpose there are minimally traumatized dental extraction techniques. The case report presented demonstrates the treatment performed in a patient who underwent extraction from unit 15 with the objective of further implant rehabilitation. The extraction was performed with the periotome, which is instrumental characteristic of being atraumatic. Dym \& Weiss, ${ }^{18}$ ratify that the periotome acts by removing the periodontal ligament (LP) from the tooth, and is placed in the gingival sulcus between the dental element and the periodontal ligament, bypassing the entire circumference of the dental element, which allows a dislocation. Without injuring the alveolar bone tables, which confirms to be considered atraumatic. ${ }^{17}$

However, in a systematic literature review, the authors suggested that although minimally invasive techniques used in dental extraction decrease the dimensional changes of the alveolar store, they could not preclude the occurrence of physiological bone resorption after extraction. ${ }^{19.20}$ Smieszek-Wilczewska et al., ${ }^{21}$ through research comparing the Bio-gen ${ }^{\circledR}$ equine graft with the Bio-oss ${ }^{\circledR}$ cattle, found that the former showed a statistically significant increase in radiological density compared to other biomaterials confirming the efficiency of the equine biomaterial. ${ }^{21}$

One of the differences of the equine graft is related to its porosity. Zielak et al. ${ }^{22}$ showed that the original bone coming from the horse, that is, equine when compared to the bovine and ovine origin was the one that presented the closest resemblance to human bone tissue, in relation to visual roughness, as well as related to porosity. . The presence of this porosity, the conformation, the diameter, the presence of intercommunication, as well as the number of pores are both directly related to migration and cellular vascularization, a fact that positively influences bone repair. ${ }^{22}$

The xenogen graft of equine origin used in this case presented an efficient bone neoformation. In a short period of radiographic evaluations, it was observed that there was satisfactory bone regeneration. These observed data corroborate the results of 
Di Stefano et al., ${ }^{11}$ who in search of more evidence about the effectiveness of equine xenograft conducted a clinical, histological and immunohistochemical study in which the objective was to evaluate the adequacy of this new equine spongy bone graft. In conclusion, to the results of this study reported that the equine graft material used is biocompatible and is associated with new blood vessels in growth during healing, a fact of great importance in bone formation. Through the study sample, they found that equine material can be safely and successfully used to increase mandibular ridges. ${ }^{11}$

In the clinical case reported, the xenogen graft was used to fill the socket, so that in a second surgical stage the installation with osseointegrated implants was performed. Di Stefano et al. conducted more research with equine xenograft, and the most recent in 2017, evaluating the use of equine matrix in combination with equine membrane to increase bone thickness in the anterior maxilla with a view to subsequent implant lateral incisor region. After follow-up through computed tomography images, they demonstrated that bone growth in the peri-implant regions and rim thickness were maintained, with alveolar crest remodeling and this indicates that the use of equine membrane and graft is a very viable option for implant-supported prosthetic rehabilitation in the aesthetic region..$^{23}$

\section{Conclusion}

The 90-day radiographic follow-up showed that the new model of xenograft (equine) has relevant characteristics, good acceptance and rapid bone neoformation, which makes possible the installation of a posterior dental implant. With a good new formation. However, further research should be performed with computed tomography (CT) to better evaluate the efficacy of equine xenograft.

\section{Funding}

None.

\section{Acknowledgments}

None.

\section{Conflict of interest}

The author declares that there is no conflict of interest.

\section{References}

1. Chappuis V, Engel O, Reyes M, et al. Ridge Alterations Postextraction in the Esthetic Zone: A 3D Analysis with CBCT. J Dent Res. 2013;92(12 Suppl):195S-201S

2. Misawa M, Lindhe J, Araújo Mg. The alveolar process following singletooth extraction: a study of maxillary incisor and premolar sites in man. Clin Oral Implants Res. 2016;27(7):884-889.

3. Sanz ME, Vignoletti F. Key aspects on the use of bone substitutes for bone regeneration of edentulous ridges. Dental Materials. 2015;31(6):640-647.

4. Araújo ME, Lindhe J. The Edentulous Ridge. In: Lindhe J, Lang NP, Karring T, editors. Clinical Periodontology and Implant Dentistry. $5^{\text {th }}$ ed. Oxford: John Wiley e Sons, Ltd; 2015. pp. 65-80.

5. Tomm A, Mezzomo RJ. Guided Bone Regeneration In Aesthetics Literature Review. Journal of Oral Investigations. 2017;6(2):62-73.
6. Dinato JC, Nunes LSS, Smidt R. Surgical techniques for bone regeneration enabling the installation of implants. In: Eduardo Saba-Chufji, Silvio Antonio dos Santos Pereira, editors. Periodontology: integration and results. Sao Paulo; Medical Arts;2007:183-226.

7. Oliveira SM, Mijares DQ, Turner G, et al. Engineering endochondral bone: in vivo studies. Tissue Eng Part A. 2009;15(3):635-643.

8. Fardin AC, Jardim ECG, Pereira FC, et al. Bone graft in dentistry: review of literature. Innovations Implant Journal. Biomaterials and Esthetics. 2010;5(3):48-52.

9. Calasans-maia M, Fernandes G, Granjeiro J. Preservation of the alveolar borderextraction for implantology. Implant News. 2008;5(6):583-590.

10. Baldini N, De sanctis M, Ferrari M. Deproteinized bovine bone in periodontal and implant surgery. Dental Materials. 2011;27(1):61-70.

11. Di Stefano DA, Aterse L, Iezzi G, et al. Alveolar ridge regeneration with equine spongy bone: a clinical, histological, and immunohistochemical case series. Clin Implant Dental relat res. 2009;11(2):90-100.

12. Di Stefano DA, Gastaldi G, Vinci R, et al. Bone formation following sinus augmentation with an equine-derived bone graft: a retrospective histologic and histomorphometric study with 36-month follow-up. Int $J$ Oral Maxillofac Implants. 2016;31(2):406-412.

13. Wang RE, Lang NP. Ridge preservation after tooth extraction. Clin Oral Implants Res. 2012;23 Suppl 6:147-156.

14. Horowitz R, Holtzclaw D, Rosen PS. A review on alveolar ridge preservation following tooth extraction. J Evid Based Dent Pract. 2012;12(3):149-60.

15. Thalmair T, Fickl S, Schneider D, et al. Dimensional alterations of extraction sites after different alveolar ridge preservation techniques - a volumetric study. J Clin Periodontol. 2013;40(7):721-727.

16. Morjaria KR, Wilson R, Palmer RM. Bone healing after tooth extraction with or without an intervention: a systematic review of randomized controlled trials. Clin Implant Dent Relat Res. 2014;16(1):1-20.

17. Zafiropoulos G. Immediate implant placement in fresh mandibular molar extraction socket: 8-year results. A case report. J Oral Implantol Abingdon. 2010;2(36):145-151.

18. Dym H, Weiss A. Exodontia: Tips and Techniques for Better Outcomes. Dent Clin N Am. 2012;56(1):245-266.

19. Ten Heggeler JM, Slot DE, Van der Weijden GA. Effect of socket preservation therapies following tooth extraction in non-molar regions in humans: A systematic review. Clin Oral Implantss Res. 2011;22(8):779788.

20. Vignoletti F, Matesanz P, Rodrigo D, et al. Surgical protocols for ridge preservation after tooth extraction. A systemic review. Clin Oral Implants Res. 2012;23(suppl):S22-S38.

21. Smieszek-wilczewsa J, Koszowski R, Pajak J. Comparison of postoperation bone defects healing of alveolar processes of maxilla and mandible with the use of bio-gen and bio-oss. J Clin Exp Dent. 2010;2(2):62-68.

22. Zielak JC. Avaliação ultra estrutural comparativa de biomaterial particulado de origem ovina. RSBO. 2011;8(1):40-47.

23. Di Stefano DA, Garagiolaa U, Bassi MA. Preserving the bone profile in anterior maxilla using an equine cortical bone membrane and an equine enzyme-treated bone graft: a case report with 5-year follow-up. J Contemp Dent Pract. 2017;18(7):614-621. 